\section{Vol. 66, No. 48}

In the report "Progress Toward Global Eradication of Dracunculiasis, January 2016-June 2017," on page 1328, the last sentence of the third paragraph should have read "The $\mathbf{1 4}$ Dracunculus specimens came from four baboons and nine dogs from Ethiopia and one dog from Chad."

On page 1331, under "Discussion," the first sentence of the second paragraph should have read "Additional interventions, including increased use of temephos and trials of potential antihelminthic treatments for infected dogs, are beginning or underway in Chad."

\section{Vol. 66, No. 50}

In the report "CDC Grand Rounds: National Amyotrophic Lateral Sclerosis (ALS) Registry Impact, Challenges, and Future Directions," on page 1382, the list of author affiliations should have read as follows:

${ }^{1}$ Environmental Health and Surveillance Branch, Division of Toxicology and Human Health Sciences, Agency for Toxic Substances and Disease Registry, CDC; ${ }^{2}$ Cynthia Shaw Crispen Chair, ALS Research, Department of Neurology, Lexington, University of Kentucky; ${ }^{3}$ person living with ALS; ${ }^{4}$ Office of the Associate Director for Science, CDC; ${ }^{5}$ Office of the Associate Director for Communication, CDC. 